\title{
Caracterização das diferenças no padrão de mobilidade de mulheres e homens em grandes cidades brasileiras
}

\section{Characterization of differences between the mobility patterns of women and men in large Brazilian cities}

\section{Bianca Macêdoํㅜ, Davi Garcia Lopes Pinto², Matheus Fontenelle Siqueira ${ }^{3}$, André Soares Lopes ${ }^{4}$, Carlos Felipe Grangeiro Loureiro ${ }^{5}$}

1Universidade Federal do Ceará, Ceará - Brasil, biancamacedo.pmf@gmail.com

${ }^{2}$ Universidade Federal do Ceará, Ceará - Brasil, davi.garcia@det.ufc.br

3Universidade Federal do Ceará, Ceará - Brasil, matheusfs@det.ufc.br

4Universidade de Fortaleza, Ceará - Brasil, soareslopes@unifor.br

5Universidade Federal do Ceará, Ceará - Brasil, felipe@det.ufc.br

\section{Recebido:}

4 de junho de 2020

Aceito para publicação:

13 de outubro de 2020

Publicado:

16 de novembro de 2020

Editor de área:

Cira Pitombo

\section{Palavras-chaves:}

Desigualdades de gênero.

Divisão sexual do trabalho.

Padrões de mobilidade urbana.

Cidades brasileiras.

\section{Keywords:}

Gender-based inequalities.

Sexual division of labor.

Urban mobility patterns.

Brazilian cities.

DOI:10.14295/transportes.v28i4.2410

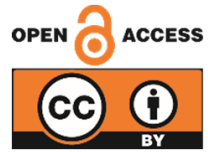

\begin{abstract}
RESUMO
Estudos em todo o mundo apontam para a ocorrência de padrões de mobilidade distintos entre mulheres e homens, que são explicados, em grande parte, pela divisão sexual do trabalho. Este esforço de pesquisa objetivou caracterizar essas diferenças para a realidade urbana brasileira. Para isso, inicialmente foram levantadas hipóteses acerca das desigualdades na mobilidade por gênero e sua relação com a divisão sexual do trabalho. Para cada hipótese levantada, indicadores foram definidos e estimados com base nos mais recentes dados de pesquisas OD domiciliares de três capitais brasileiras, representando regiões distintas do país. Os resultados obtidos para cada capital foram então comparados e discutidos, assim como analisada a evolução temporal das desigualdades na mobilidade por gênero em São Paulo, entre 2007 e 2017. Conclui-se que há evidências empíricas para validar a maioria das hipóteses levantadas, mostrando que de fato há diferenças nos padrões de deslocamentos de mulheres e homens no meio urbano brasileiro.
\end{abstract}

\begin{abstract}
Studies worldwide indicate differences between women's and men's mobility patterns, mainly caused by the sexual division of labor. This research effort intends to characterize these differences for the Brazilian urban scenario. For that, first, hypotheses were established regarding gender-based mobility inequalities and their relation to the sexual division of labor. For each stated hypothesis, indicators were defined and calculated. To do so, this research used the most recent home-based O-D survey data from three capitals of distinct Brazilian regions. In the end, we present the results for each capital and discuss the temporal evolution of gender-based mobility inequalities in São Paulo, between 2007 and 2017. The results confirm most of the hypotheses, showing that there are indeed differences in women's and men's travel patterns within the Brazilian urban environment.
\end{abstract}

\section{INTRODUCÃO}

0 gênero tem sido apontado pela comunidade científica como um fator preponderante na diferenciação de necessidades de deslocamentos. Estudos apontam que a mobilidade é diretamente influenciada pelas distintas atividades atribuídas a mulheres e homens na sociedade 
(Hanson, 2010; Kergoat, 2004; Rosenbloom, 1978; Rosenbloom e Pleiss-Fraissard, 2010). Análises de padrões de mobilidade por gênero têm evidenciado a existência de diferenças em termos de distâncias, durações, horários, motivos, modos de transporte e outras características das viagens de mulheres e homens, tanto em países do Norte Global (Crane, 2007; Hanson e Johnston, 1985; Root e Schinder, 1999; Rosenbloom, 2000, 2006; Sermons e Koppelman, 2001; Prashker et al., 2008), como do Sul Global (Lee e McDonald, 2003; Peters, 2002; Anand e Tiwari, 2006; Srinivasan e Rogers, 2005; Venter et al., 2007; Svab, 2016).

No Brasil, destaca-se o estudo de Svab (2016) que apresenta a evolução dos padrões de deslocamento de mulheres e homens na Região Metropolitana de São Paulo a partir das pesquisas OD domiciliares do metrô para os anos de 1977, 1987, 1997 e 2007. Entretanto, como o referido estudo se limita a apenas uma capital brasileira, reconhece-se uma lacuna de conhecimento decorrente da necessidade de se avaliar a generalização e a abrangência da problemática apresentada em outras grandes cidades brasileiras, especialmente de outros contextos regionais. Este estudo busca, portanto, contribuir com o conhecimento científico e técnico a partir de análises empíricas dos dados de mobilidade referentes a cidades representativas de três regiões do Brasil (Nordeste, Sudeste e Sul), expandindo-se a compreensão desse fenômeno urbano brasileiro, contexto ainda pouco abordado nas pesquisas voltadas a esse tema.

0 presente trabalho tem como objetivo geral caracterizar as diferenças no padrão de mobilidade de mulheres e homens em três grandes cidades brasileiras, representativas de diversas regiões. Este objetivo é alcançado inicialmente a partir da revisão de literatura acerca de fatores que influenciam as diferenças de mobilidade entre mulheres e homens, e em que consistem tais diferenças, de forma a embasar a seleção de indicadores para a análise (Seção 2). Em seguida, a metodologia de análise é apresentada, incluindo a definição dos dados utilizados e das cidades analisadas, bem como a descrição e justificativa dos indicadores adotados (Seção 3). Na Seção 4, realiza-se a análise comparativa por gênero dos resultados obtidos para cada indicador nas cidades selecionadas. A Seção 5 discute a evolução dessa problemática na cidade de São Paulo com a comparação dos indicadores entre 2007 e 2017. Por fim, as conclusões e recomendações deste trabalho são apresentadas na Seção 6.

\section{POR QUE E EM QUE A MOBILIDADE SE DIFERENCIA POR GÊNERO?}

Uma relevante questão social que impacta na mobilidade de mulheres e homens está relacionada ao mercado de trabalho. Kergoat (2004) afirma que existe uma divisão sexual do trabalho, caracterizada principalmente pela atribuição da esfera produtiva aos homens e da esfera reprodutiva às mulheres. Tal divisão está alicerçada nos princípios da separação (há "trabalhos de homens" e "trabalhos de mulheres") e da hierarquização (o trabalho produtivo é mais valioso que o reprodutivo). Essa designação histórica ainda possui reflexos na inserção da mulher no mercado produtivo e na sua carga de trabalho, uma vez que ela detém a maior parte das atribuições de tarefas do lar.

Esses aspectos se materializam em uma menor taxa de participação das mulheres no mercado de trabalho, que é calculada através do quociente entre a população economicamente ativa e a população total. Segundo dados de Pinheiro et al. (2009), a taxa de participação das mulheres com mais de 15 anos, em 2009, era de 68,9\%, enquanto a de homens era de 81,6\%. Esse indicador, entretanto, não reflete as características de desemprego dos grupos de interesse. Para isso, a taxa de desocupação é o indicador mais adequado, já que mede a proporção de 
pessoas desempregadas e em busca de emprego, dentre a população economicamente ativa. Em 2009, a taxa de desocupação das mulheres era de 10,2\%, contra 6\% dos homens.

As diferenças relacionadas à empregabilidade acabam se desdobrando em duas consequências. A primeira é a relação que se faz entre desemprego e imobilidade, dado o contexto de que grande parte dos movimentos capturados por pesquisas OD domiciliares são pelos motivos trabalho e estudo. A segunda, e mais clara, é a desigualdade de renda entre os dois gêneros. De acordo com o mesmo estudo de Pinheiro et al. (2009), o rendimento médio mensal no trabalho principal das mulheres correspondia a $66 \%$ do equivalente para os homens. Essa diferença é corroborada quando se verifica que a jornada semanal do trabalho principal para os homens era, em média, de 42,9 horas, enquanto para as mulheres essa média caía para 35,6 horas. Esses dados apontam para a interpretação de que há uma maior participação de mulheres em trabalhos de meio expediente. Quando incluído o trabalho doméstico não remunerado, a jornada semanal média das mulheres passou a ser de 55,3 horas, contra 47,7 dos homens. Isso reforça o caráter reprodutivo das mulheres nessa divisão de trabalho.

Pode-se perceber, portanto, que apesar dos avanços na inserção da mulher no mercado de trabalho, este ainda é marcado pela existência de desigualdades de gênero. Tais diferenças, especialmente aquelas relacionadas aos papéis domésticos, empregabilidade, ocupação e renda, acabam refletindo em padrões de mobilidade distintos entre homens e mulheres (Bowman e Ben-akiva, 1996; Rosenbloom, 2006; Sermons e Koppelman, 2001). Vale destacar, ainda, que outros fatores, como os relacionados à segurança pública (crimes, assaltos, furtos, violência e assédio sexual, por exemplo), que constituem um considerável problema para mulheres nos países em desenvolvimento (Loukaitou-Sideris, 2010), também proporcionam diferenças nesses padrões de mobilidade. No entanto, tais aspectos não são foco do presente estudo, já que se optou por analisar padrões de mobilidade afetados apenas pela divisão sexual do trabalho.

\subsection{Estudos anteriores sobre as diferenças na mobilidade entre os gêneros}

Alguns estudos já buscaram caracterizar as diferenças nos padrões de mobilidade de homens e mulheres a partir das desigualdades na divisão sexual do trabalho. Foram identificados trabalhos em diversas regiões do globo, os quais refletem a realidade tanto de países desenvolvidos quanto daqueles emergentes. Observou-se, nos estudos analisados, resultados muito semelhantes em termos das diferenças nos comportamentos de viagem de mulheres e homens (Law, 1999; Rosenbloom e Maryvonne Pleiss-Fraissard, 2010; Peters, 2002). Ao longo desta subseção, busca-se apresentar, de forma breve, o estado de compreensão mais recente sobre as distinções nos padrões de mobilidade entre os gêneros.

A menor inserção das mulheres no mercado de trabalho, aliada às atribuídas responsabilidades de trabalho doméstico, implica em comportamentos de mobilidade mais complexos para o gênero feminino. Como as mulheres tendem a realizar mais viagens por outros motivos que não sejam trabalho, há uma tendência da realização de uma maior quantidade de viagens do que os homens, porém com menores distâncias (Crane, 2007; Hanson e Johnston, 1985; Rosenbloom, 2000; Svab, 2016). Essas viagens são, assim, mais encadeadas do que baseadas no domicílio, de forma a combinar as diversas necessidades de deslocamento em únicas jornadas para atender as limitações de disponibilidade de tempo (Root e Schinder, 1999). Além disso, a maior variedade de motivos de viagem faz com que as mulheres possuam uma maior diversidade de origens e destinos, além de uma maior proporção de viagens acontecendo em períodos fora do pico (Rosenbloom e Maryvonne Pleiss-Fraissard, 2010). 
Além de realizarem viagens relativas às próprias necessidades, as mulheres também se encarregam das viagens relacionadas à provisão de alimentos para o lar e ao acompanhamento de idosos para suas respectivas atividades (consulta médica, por exemplo). Além disso, elas possuem padrão de mobilidade mais afetado pela presença de crianças no domicílio (Root e Schinder, 1999; Rosenbloom, 2006; Srinivasan e Rogers, 2005; Svab, 2016). Decorre disso, portanto, que as mulheres tendem a realizar menos viagens por motivo trabalho e mais viagens por outros motivos (compras, saúde, educação, servir passageiro), se comparadas aos homens. Observa-se, ainda, diferenças por gênero na divisão modal das viagens. Constata-se que as mulheres tendem a utilizar mais o transporte público do que os homens, além de realizarem mais viagens a pé (Anand e Tiwari, 2006; Crane, 2007; Root e Schinder, 1999; Rosenbloom, 2000; Srinivasan e Rogers, 2005; Svab, 2016). Isso pode ser atribuído ao fato de mulheres possuírem, em média, uma renda mensal menor do que a dos homens, dificultando assim o acesso à posse e ao uso de veículos (carros, motos e bicicletas). Por conseguinte, as mulheres tendem a passar mais tempo deslocando-se ao longo do dia, dado que fazem maior número de viagens utilizando meios de transporte de menor velocidade operacional (Anand e Tiwari, 2006; Prashker et al., 2008; Rosenbloom, 2006; Svab, 2016; Venter et al., 2007).

\subsection{Hipóteses de estudo}

Com base na revisão acima, a Figura 1 apresenta uma proposta de representação da problemática em estudo. São apresentadas hipóteses de relações de causalidade entre a divisão sexual do trabalho e os distintos padrões de mobilidade de homens e mulheres, buscando explicar em que diferem tais padrões. As caixas inferiores representam as hipóteses relacionadas às diferenças de padrões de mobilidade por gênero, as quais serão caracterizadas nas seções seguintes. Já as caixas superiores referem-se à divisão sexual do trabalho, sendo tratadas aqui como premissas que explicam a existência das diferenças em mobilidade.

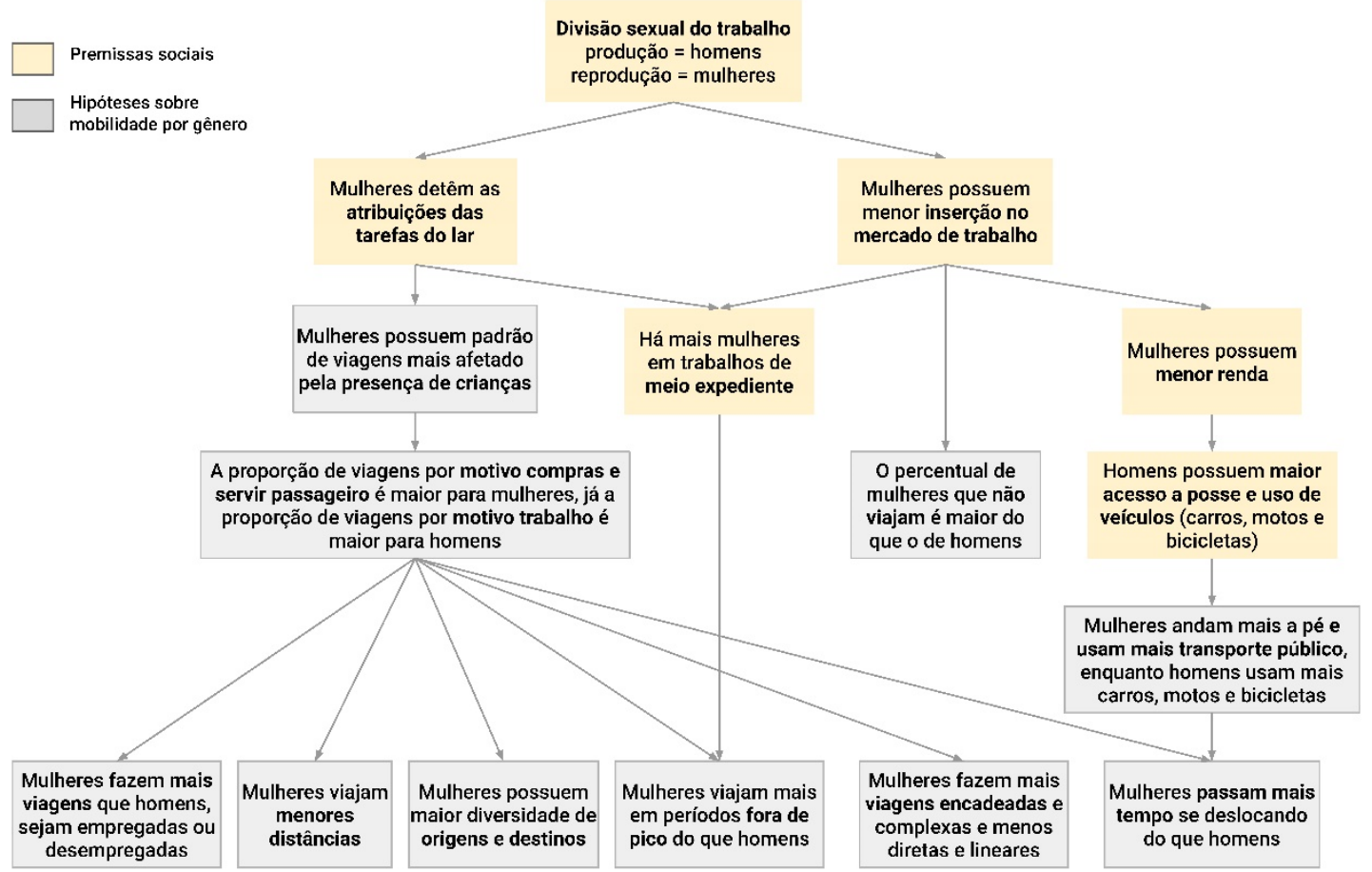

Figura 1. Hipóteses de relações entre as diferenças de padrões de mobilidade por gênero e a divisão sexual do trabalho 


\section{PROPOSTA METODOLÓGICA DE CARACTERIZAÇÃO}

Nesta seção, apresenta-se um método de caracterização para a problemática da desigualdade nos padrões de mobilidade por gênero. 0 foco é investigar as hipóteses levantadas na seção anterior. A proposta metodológica se baseia, inicialmente, na seleção das cidades brasileiras que serão analisadas ao longo do estudo e nas bases de dados utilizadas. Em seguida, realiza-se a definição de indicadores para cada hipótese apresentada, com o objetivo de quantificar as diferenças entre homens e mulheres. Vale destacar que não faz parte do escopo desse trabalho realizar análise de diagnóstico das relações de causalidade propostas.

\subsection{Seleção das cidades de estudo e bases de dados}

As análises empíricas foram conduzidas a partir de pesquisas de origem-destino (OD) domiciliares, que constituem a principal fonte de informações sobre os padrões de mobilidade da população brasileira nas grandes cidades, englobando informações sobre as características dos indivíduos, das famílias e dos domicílios. Dentre as capitais que realizaram pesquisas OD domiciliares na última década, foram escolhidas cidades de diferentes regiões do país que pudessem refletir os distintos contextos socioeconômicos nacionais. Além disso, julgou-se fundamental a escolha de pesquisas domiciliares mais recentes, de modo a trazer uma caracterização mais representativa da situação urbana atual vivenciada por brasileiras e brasileiros. Com base nesses critérios, definiu-se as cidades de São Paulo, Salvador e Florianópolis como locais de estudo para as análises (Tabela 1).

Tabela 1 - Pesquisas de origem-destino selecionadas para as análises

\begin{tabular}{cccc}
\hline Região Metropolitana & São Paulo & Salvador & Florianópolis \\
\hline Ano da pesquisa mais recente & 2017 & 2012 & 2014 \\
Domicílios pesquisados & $32 \mathrm{mil}$ & $20 \mathrm{mil}$ & $5 \mathrm{mil}$ \\
Indivíduos entrevistados & $86 \mathrm{mil}$ & $59 \mathrm{mil}$ & $12 \mathrm{mil}$ \\
Viagens registradas & $183 \mathrm{mil}$ & $96 \mathrm{mil}$ & $20,8 \mathrm{mil}$ \\
\hline
\end{tabular}

\subsection{Definição dos indicadores}

Buscou-se selecionar indicadores já bem estabelecidos nos trabalhos acadêmicos sobre desigualdades na mobilidade por gênero, que foram a principal fonte de informação para a revisão de literatura, embasando a elaboração das hipóteses de estudo. Entretanto, o estabelecimento dos indicadores também foi determinado pela estrutura e disponibilidade dos dados constantes nas pesquisas OD analisadas. Observou-se que, apesar de haver uma estrutura básica comum entre os bancos de dados, há disparidades na definição dos motivos de viagem e na coleta de informações sobre a estrutura familiar (responsável, marido/esposa, filhos, etc.). Além disso, nem sempre há informações a respeito da situação empregatícia dos indivíduos. A Tabela 2 lista os indicadores definidos para mensurar cada uma das hipóteses aqui levantadas acerca das desigualdades por gênero na mobilidade urbana.

Todos os indicadores foram estimados para os dois segmentos analisados (mulheres e homens), permitindo assim a apresentação das análises de maneira comparativa. Alguns comentários valem ser destacados. 0 indicador (1) refere-se às taxas de imobilidade da população, enquanto o indicador (2) consiste na taxa média de viagens dos indivíduos que realizaram alguma viagem, diferindo das taxas de mobilidade usualmente calculadas que consideram o total 
de viagens realizadas e o total de indivíduos entrevistados. 0 conceito de imobilidade ("não realizar viagens") corresponde aos indivíduos que não possuem viagens registradas no banco de dados da pesquisa OD, seja porque não reportaram viagens, ou porque a viagem foi realizada a pé a uma distância curta de menos de $500 \mathrm{~m}$. Foi considerado, ainda, que a possível existência de viagens não reportadas pelos usuários no momento da entrevista, fenômeno denominado de "soft refusal" (Motte-Baumvol e Bonin, 2018), é aleatória e não traz viés aos resultados. Nas estimativas desses indicadores, foram consideradas apenas pessoas com idade a partir de 15 anos, consideradas economicamente ativas de acordo com a PNAD (Pesquisa Nacional de Amostra por Domicílios) contínua.

Tabela 2 - Desigualdades por gênero na mobilidade: hipóteses e indicadores propostos

\begin{tabular}{|c|c|c|}
\hline HIPÓTESE & & INDICADOR \\
\hline $\begin{array}{l}\text { A proporção de mulheres que não realizam viagens é } \\
\text { maior do que a dos homens }\end{array}$ & (1) & $\begin{array}{l}\text { Percentual de pessoas, com idade maior ou igual a } 15 \text { anos, } \\
\text { que não realizam viagens }\end{array}$ \\
\hline Mulheres fazem mais viagens que homens & $(2)$ & $\begin{array}{l}\text { Número médio de viagens por pessoa, com } 15 \text { anos ou mais, } \\
\text { dado que esta realizou alguma viagem }\end{array}$ \\
\hline $\begin{array}{l}\text { Mulheres andam mais a pé e usam o transporte público, } \\
\text { enquanto os homens usam mais carros, motos e } \\
\text { bicicletas }\end{array}$ & (3) & $\begin{array}{l}\text { Proporção de cada modo, por gênero, incluindo transporte } \\
\text { público, a pé, bicicleta, carro (dirigindo e passageiro), moto e } \\
\text { outros }\end{array}$ \\
\hline $\begin{array}{l}\text { A proporção de viagens por motivo compras e servir } \\
\text { passageiro é maior para mulheres, enquanto por motivo } \\
\text { trabalho é maior para homens }\end{array}$ & (4) & $\begin{array}{l}\text { Percentual de cada motivo, por gênero, incluindo os motivos } \\
\text { trabalho, estudo, compras, servir passageiro e outros }\end{array}$ \\
\hline Mulheres viajam menores distâncias & $(5)$ & Distância de viagem por gênero \\
\hline $\begin{array}{l}\text { Mulheres viajam mais em períodos fora de pico do que } \\
\text { homens }\end{array}$ & (6) & Percentual de viagens, por gênero, no pico e no fora pico \\
\hline $\begin{array}{l}\text { Mulheres passam mais tempo se deslocando do que } \\
\text { homens }\end{array}$ & (7) & $\begin{array}{l}\text { Média do somatório dos tempos de viagem ao longo de um } \\
\text { dia por gênero }\end{array}$ \\
\hline $\begin{array}{l}\text { Mulheres fazem mais viagens encadeadas e complexas e } \\
\text { menos diretas e lineares }\end{array}$ & (8) & Proporção de viagens de base não-residencial por gênero \\
\hline $\begin{array}{l}\text { Mulheres possuem padrão de viagens mais afetado pela } \\
\text { presença de crianças }\end{array}$ & (9) & $\begin{array}{l}\text { (Análise dos indicadores anteriores estratificados pela } \\
\text { presença ou ausência de crianças na família) }\end{array}$ \\
\hline
\end{tabular}

Os indicadores (3) e (4) baseiam-se na repartição modal e na distribuição dos motivos das viagens, respectivamente. Os modos considerados foram: público, a pé, bicicleta, automóvel (dirigindo ou passageiro), motocicleta e outros. Os motivos de viagem considerados foram cinco: trabalho, estudo, compras, servir passageiro e outros. Para o indicador (5), utilizou-se as distâncias euclidianas em quilômetros. Apesar das limitações relativas ao uso dessas distâncias aproximadas, considera-se que em regiões com malha viária densa, como é o caso dos locais analisados, o impacto dessa aproximação tende a ser reduzido.

\section{RESULTADOS}

A seguir, são apresentados os resultados dos indicadores para as três capitais analisadas. Em cada subseção serão analisadas as hipóteses estabelecidas anteriormente, considerando estimativas com margens de erro inferiores a $2 \%$ para as proporções populacionais, com $95 \%$ de grau de confiança.

\subsection{Diferenças na imobilidade}

De acordo com as pesquisas analisadas, a imobilidade é consideravelmente superior entre as 
mulheres (Figura 2), abrangendo cerca de 1/3 dos indivíduos desse gênero em São Paulo e Salvador, e cerca de $1 / 4$ das mulheres em Florianópolis. Sendo a mobilidade inerente à necessidade dos indivíduos de acessarem atividades e oportunidades, os resultados observados podem ser explicados pela tendência da atribuição às mulheres das tarefas do lar, que em sua maioria independem da vivência do meio urbano. A maior imobilidade de mulheres, mostrada nas pesquisas, pode ser consequência, ainda, da realização de deslocamentos curtos, inferiores a 500 metros, que tendem a não ser registrados nas pesquisas OD aplicadas no Brasil.

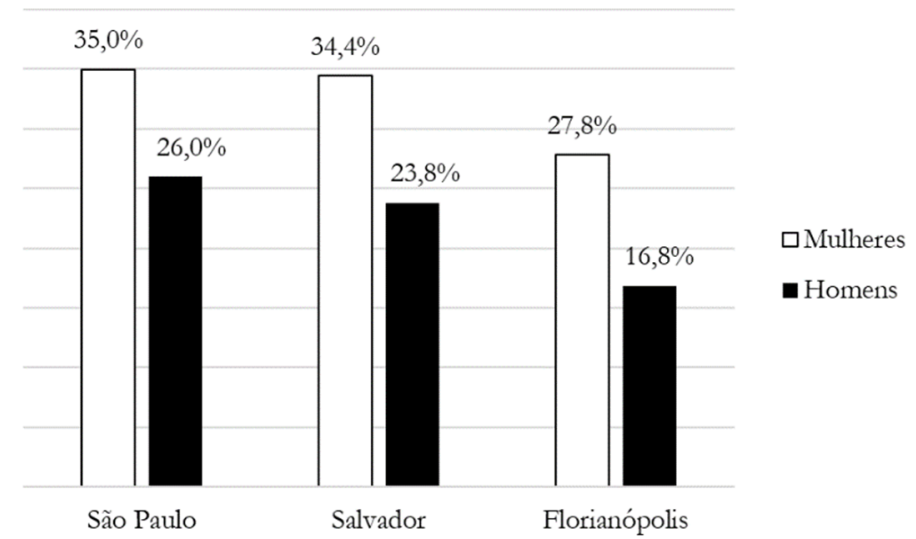

Figura 2. Taxas de imobilidade de mulheres e homens nas três capitais

\subsection{Mobilidade de indivíduos ativos}

Apesar da maior taxa de imobilidade das mulheres, quando se analisam apenas os indivíduos ativos (a partir de 15 anos de idade) que relataram deslocamentos, verifica-se, pela Figura 3, que não há diferenças significativas na quantidade média de viagens de mulheres e homens. Duas situações opostas podem contribuir para tal fato: a menor inserção das mulheres no mercado de trabalho, que tende a reduzir a quantidade de viagens por esse motivo; e a necessidade das mulheres em realizar deslocamentos ligados às necessidades pessoais e do lar, a qual tenderia ao aumento dessa média.

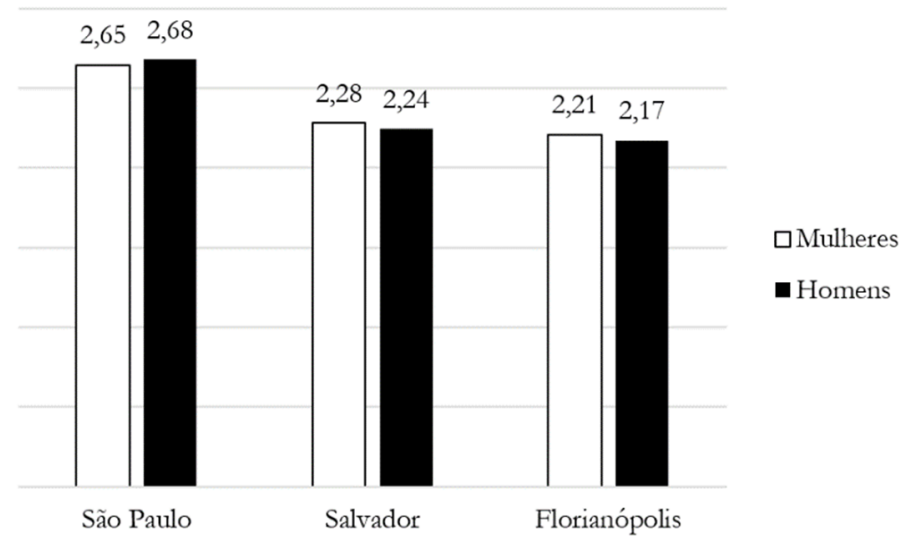

Figura 3. Quantidade média de viagens realizadas por mulheres e homens ativos, com deslocamentos reportados nas três capitais

\subsection{Repartição modal}

A análise da repartição das viagens observadas (Figura 4) tende a corroborar as hipóteses estabelecidas a priori. Em relação aos modos ativos, verifica-se uma tendência das mulheres em andar mais a pé do que os homens. Essa tendência, entretanto, inverte-se no uso de bicicleta. 
Com relação ao uso de modos motorizados individuais, observa-se a preponderância dos indivíduos do gênero masculino. Isso se reflete na tendência de as mulheres utilizarem mais o automóvel como passageiras e menos como motoristas, quando comparadas aos homens. Essas discrepâncias apontam para padrões de viagens com menor alcance (dentro de um mesmo intervalo de tempo) e, consequentemente, um menor acesso a oportunidades por parte das mulheres.

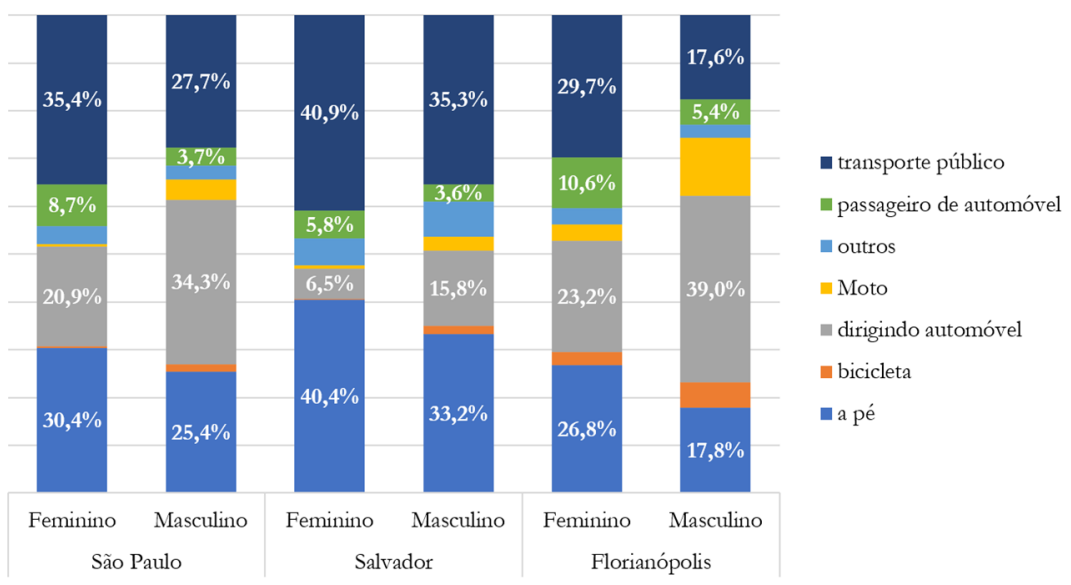

Figura 4. Divisão modal de viagens realizadas por mulheres e homens

\subsection{Motivos de viagem}

A partir da análise da distribuição dos motivos de viagem (Figura 5), percebe-se, nas três cidades analisadas, uma menor participação das mulheres em deslocamentos por motivo trabalho. Já para as viagens por motivos educacionais, foi observada uma relativa paridade entre os dois gêneros. Por fim, verifica-se, para os casos de São Paulo e Salvador, maior participação feminina nas viagens cujo motivo foi servir passageiros, corroborando a hipótese levantada acerca dessa divisão. Não foi possível, entretanto, verificar essa hipótese para a cidade de Florianópolis, pois a pesquisa lá realizada não discriminou esse motivo de viagem.

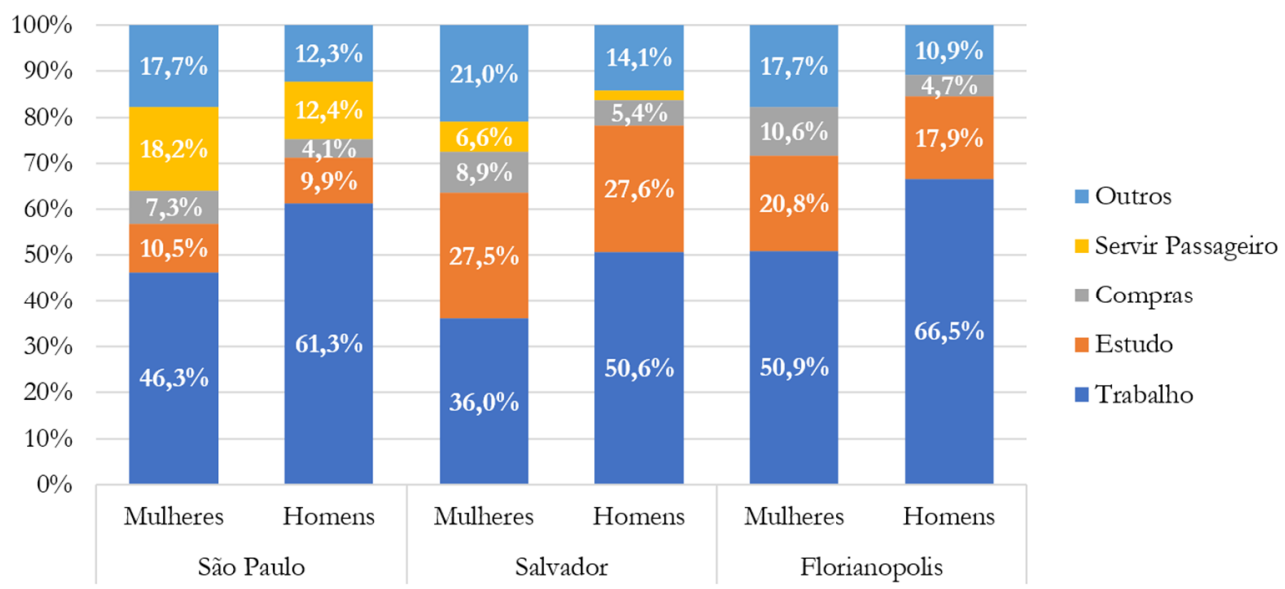

Figura 5. Motivos das viagens de mulheres e homens nas três capitais

\subsection{Distâncias de viagem}

A Figura 6 apresenta os resultados das distâncias médias das viagens realizadas. Para as três cidades analisadas, pode-se verificar a hipótese de que as mulheres se deslocam, em média, em 
menores distâncias do que os homens. Esse resultado reforça a hipótese de que as mulheres possuem menor alcance em suas viagens, principalmente em função da utilização de modos ativos (a pé e bicicleta), além do papel de cumprir necessidades cotidianas, como compras do dia a dia e caronas a familiares, que elevam as pressões nos seus orçamentos de tempo.

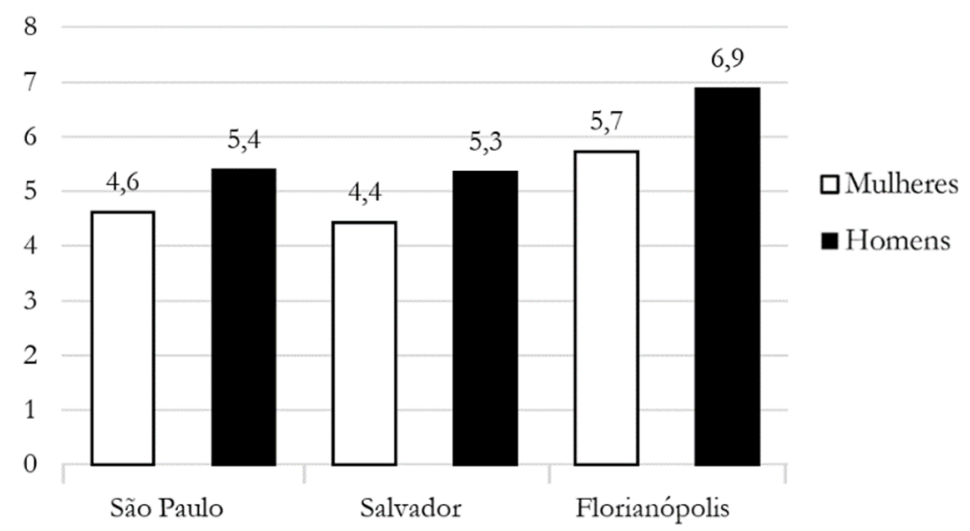

Figura 6. Distâncias euclidianas médias (em $\mathrm{km}$ ) das viagens realizadas

\subsection{Realização de viagens em horários fora de pico}

Espera-se que viagens que não sejam por motivo trabalho ou estudo estejam mais bem distribuídas ao longo do dia, devido à maior flexibilidade na execução dessas atividades. Dada a maior participação feminina em viagens desse tipo, a expectativa é, portanto, que haja uma predominância nos deslocamentos das mulheres fora dos horários de pico. Isso pode ser observado a partir dos resultados da Figura 7, principalmente nas cidades de Salvador e Florianópolis, cujas diferenças percentuais entre os gêneros foram de 4\% e 7\%, respectivamente. Já em São Paulo a diferença encontrada (1\%) não foi significativa.

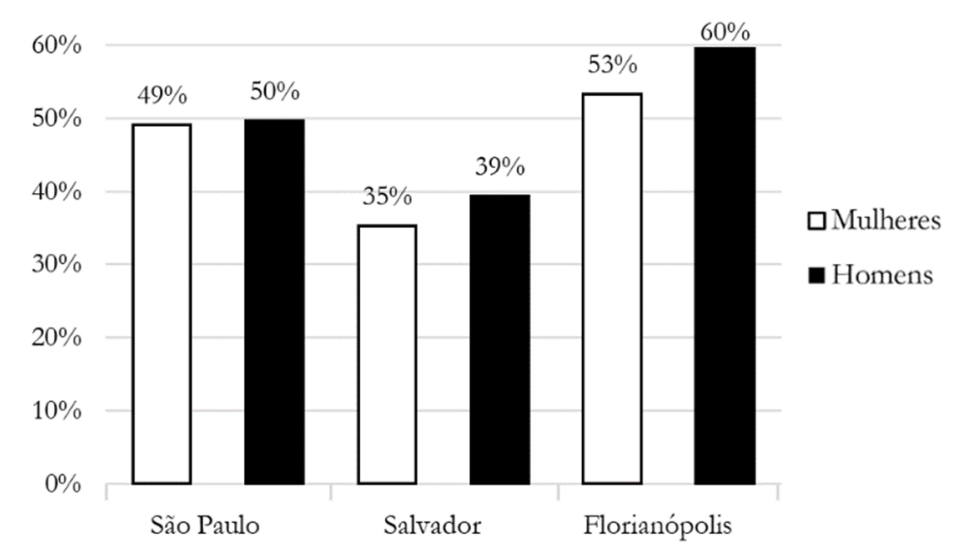

Figura 7. Proporção de viagens realizadas em horários fora de pico

\subsection{Tempo gasto diariamente em deslocamentos}

Segundo a hipótese levantada, as mulheres passam mais tempo se deslocando ao longo do dia do que os homens. A Figura 8, entretanto, aponta que essa condição só se confirma em Florianópolis. Tal constatação contradiz a argumentação de que esse maior tempo em trânsito ocorre por conta de uma maior utilização de modos menos velozes por parte das mulheres, pois é na capital sulista onde elas mais utilizam automóvel (dirigindo ou como passageiro) e apresentam os maiores percentuais de viagens compulsórias. 


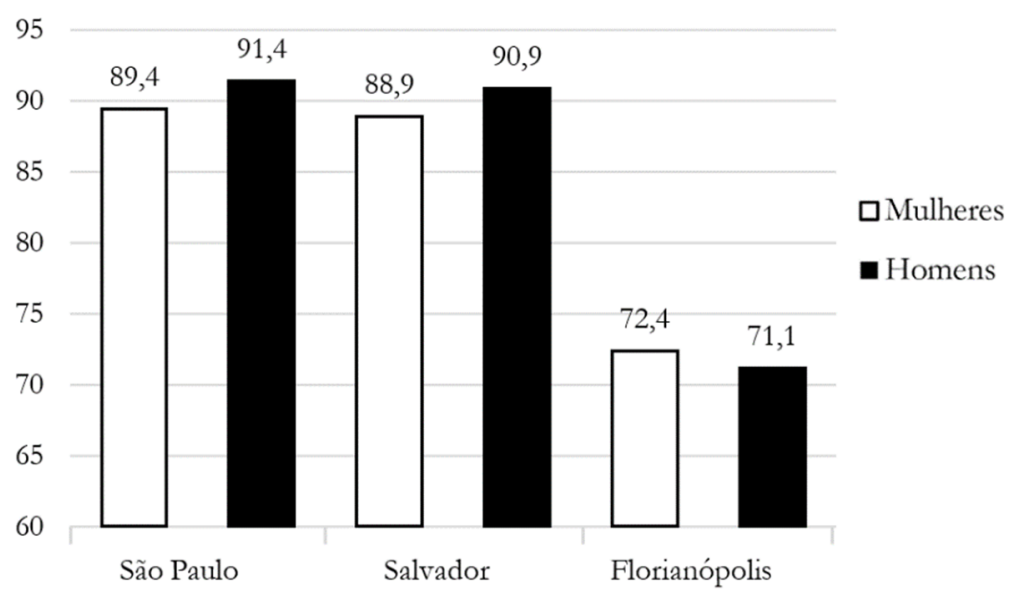

Figura 8. Tempo total, em minutos, gasto nos deslocamentos diários

\subsection{Encadeamento de viagens}

Entende-se por viagens encadeadas aquele conjunto de deslocamentos que têm motivos e pares OD associados, de modo que o destino da viagem anterior é igual à origem da viagem subsequente. Face à indisponibilidade desse dado nas pesquisas analisadas, assumiu-se, como premissa nessa análise, que a proporção de viagens de base não-domiciliar de um determinado segmento poderia representar uma proxy do encadeamento das viagens nesse grupo, já que em uma viagem encadeada apenas a primeira e a última pernas são de base domiciliar, enquanto as demais são de base não-domiciliar. Diante disso, os resultados observados na Figura 9 não constituem evidências significativas para apoiar a hipótese de que as mulheres apresentam um maior encadeamento de viagens: nem no caso de Florianópolis, onde a proporção de viagens de base não-domiciliar é ligeiramente maior para as mulheres; nem muito menos em São Paulo e Salvador, onde os homens apresentaram maior proporção de viagens cujas origens ou destinos não foram o domicílio.

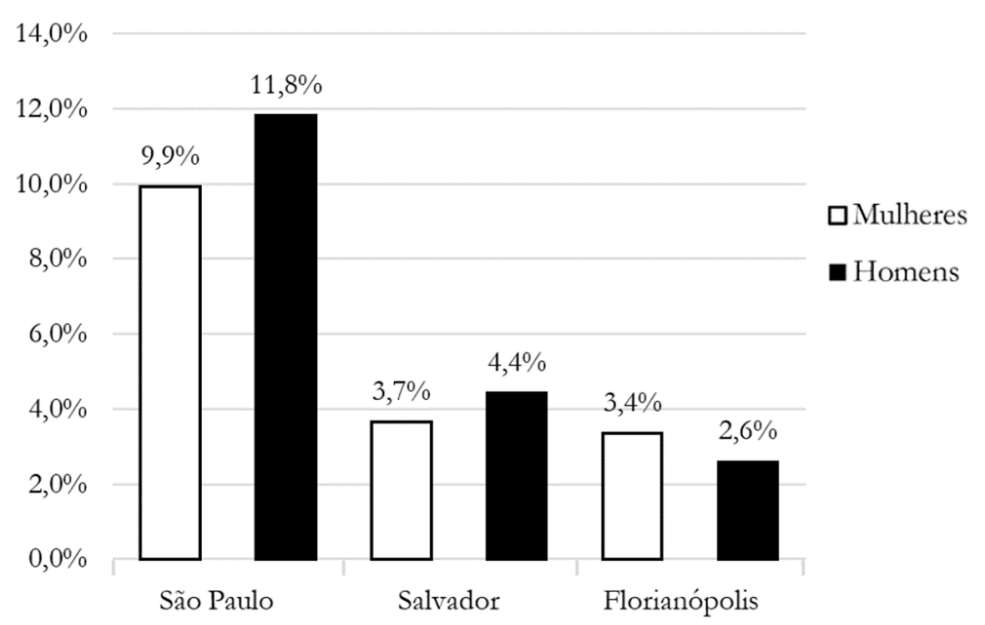

Figura 9. Proporção de viagens de base não-domiciliar por gênero

\subsection{Influência na mobilidade da presença de crianças na estrutura familiar}

Entende-se que a presença de crianças na estrutura familiar afeta, em maior grau, a mobilidade 
feminina, dado que a mulher é a maior responsável pelo acompanhamento de dependentes no acesso a atividades fora do domicílio. Os resultados apresentados na Tabela 3 reforçam essa conjectura. Observa-se que mulheres com crianças em casa possuem uma taxa de mobilidade maior, quando comparadas com a média geral. Nota-se ainda um reflexo da presença de crianças na distribuição dos motivos de viagens dessas mulheres, verificando-se um aumento da proporção de viagens para servir passageiros.

Tabela 3 - Efeitos da presença de crianças na estrutura familiar sobre a mobilidade feminina

\begin{tabular}{l|c|ccc|ccc}
\hline \multirow{2}{*}{ Indicador } & \multicolumn{3}{c|}{ Mulheres em geral } & \multicolumn{3}{c}{ Mulheres com crianças na família } \\
\cline { 2 - 8 } & SP & SALV & FL & SP & SALV & FL \\
\hline $\begin{array}{c}\text { Proporção de mulheres em idade ativa que não realizam } \\
\text { viagens (\%) }\end{array}$ & 35,0 & 34,4 & 27,8 & 30,6 & 32,9 & 27,4 \\
\hline $\begin{array}{c}\text { Número médio de viagens por pessoa em idade ativa } \\
\text { (apenas indivíduos que realizam viagens) }\end{array}$ & 2,65 & 2,28 & 2,21 & 3,00 & 2,36 & 2,22 \\
\hline & Trabalho & 46,3 & 35,1 & 50,9 & 38,0 & 28,7 & 44,4 \\
\hline
\end{tabular}

\section{DISCUSSÃO SOBRE A EVOLUÇÃO DAS DESIGUALDADES EM SÃO PAULO}

Apesar de elucidativos, os dados levantados e as análises de caracterização realizadas acima são apenas retratos estáticos das diferenças nos padrões de mobilidade entre gêneros nas três cidades estudadas. Eles são a representação das condições que as pesquisas OD domiciliares foram capazes de capturar, mas não dizem muito acerca das tendências de melhora ou piora desse contexto problemático de desigualdades. Portanto, decidiu-se por investigar também a evolução temporal dos indicadores. Para tanto, foram utilizados os dados das pesquisas domiciliares referentes aos anos de 2007 e 2017 de São Paulo, única das cidades brasileiras que possui duas pesquisas OD domiciliares realizadas, com consistência metodológica, nas duas últimas décadas. Os valores dos indicadores para os dois recortes temporais supracitados encontram-se na Tabela 4.

Aplicados os mesmos indicadores aos dois conjuntos de dados, é possível observar variações para mais e para menos de cada medida ao longo do tempo. Em resumo, proporcionalmente menos mulheres em idade ativa permanecem imóveis (não realizam viagens), ao mesmo tempo em que estas passaram a fazer menos viagens por dia, em média. Observou-se, tanto para as mulheres quanto para os homens, uma redução do uso do automóvel e crescimento do modo a pé na divisão modal. Já a utilização do transporte público cresceu dentre as mulheres e reduziu dentre os homens. Além disso, as distâncias médias de viagem feitas por mulheres vêm aumentando, assim como a proporção de viagens feitas por elas no horário de pico. Curiosamente, tanto o tempo total gasto com deslocamentos (-12,7\% e -14,9\%), quanto a proporção de viagens com base não-residencial $(-16,8 \%$ e $-4,1 \%)$, diminuíram para mulheres e homens, respectivamente.

Para se avaliar a melhora ou piora das desigualdades entre gêneros ao longo do tempo é importante não apenas fazer uma comparação temporal dos valores dos indicadores como também analisar as tendências de mudanças e a convergência desses indicadores a uma condição de menor desigualdade. Dessa forma, percebe-se uma tendência de aumento dessas diferenças para alguns indicadores em São Paulo, como é o caso da imobilidade que, em um intervalo de 
10 anos, apesar de ter diminuído para ambos os gêneros, apresentou ganhos maiores para homens do que para mulheres. 0 mesmo ocorre com as proporções de viagens no transporte público, com motivo 'servir passageiro' e de base não-domiciliar. Já para outros indicadores houve uma redução absoluta das diferenças entre gêneros, nos quais foram observados ganhos maiores para as mulheres. Esse foi o caso da proporção das viagens a pé, assim como dos indicadores de distância média e tempo total dos deslocamentos. Já os indicadores relacionados à taxa de mobilidade, uso do automóvel, motivos trabalho e compras, além da distribuição horária das viagens, em todos não houve variações significativas para a redução das desigualdades entre gêneros.

Tabela 4 - Comparação das pesquisas OD domiciliares de 2007 e 2017 de São Paulo

\begin{tabular}{|c|c|c|c|c|c|c|c|}
\hline \multirow{2}{*}{\multicolumn{2}{|c|}{ Indicador }} & \multicolumn{2}{|c|}{2007} & \multicolumn{2}{|c|}{2017} & \multirow{2}{*}{$\begin{array}{l}\text { Variação } \\
\text { absoluta das } \\
\text { diferenças }\end{array}$} & \multirow[t]{2}{*}{$\begin{array}{c}\text { Tendência de } \\
\text { Evolução da } \\
\text { Desigualdade } \\
\end{array}$} \\
\hline & & M & $\mathbf{H}$ & M & $\mathbf{H}$ & & \\
\hline \multicolumn{2}{|c|}{$\begin{array}{l}\text { Proporção de pessoas em idade ativa que } \\
\text { não realizam viagens (\%) }\end{array}$} & 35,6 & 28,2 & $35,0 \downarrow$ & $26,0 \downarrow$ & $+1,6$ & Aumentou ( $\uparrow$ ) \\
\hline \multicolumn{2}{|c|}{$\begin{array}{c}\text { Número médio de viagens por pessoa em } \\
\text { idade ativa (apenas considerando indiví- } \\
\text { duos que realizam viagens) }\end{array}$} & 2,75 & 2,71 & $2,65 \downarrow$ & $2,68 \downarrow$ & $-0,07$ & Estável ( ) \\
\hline \multirow{3}{*}{$\begin{array}{l}\text { Repartição modal } \\
(\%)\end{array}$} & A pé & 28,6 & 21,7 & $30,4 \uparrow$ & $25,4 \uparrow$ & $-1,9$ & Diminuiu $(\downarrow)$ \\
\hline & $\begin{array}{l}\text { Transporte } \\
\text { público }\end{array}$ & 33,9 & 28,4 & $35,4 \uparrow$ & $27,7 \downarrow$ & $+2,2$ & Aumentou ( $\uparrow$ ) \\
\hline & $\begin{array}{l}\text { Dirigindo } \\
\text { automóvel }\end{array}$ & 24,2 & 37,9 & $20,9 \downarrow$ & $34,3 \downarrow$ & $-0,3$ & Estável ( ) \\
\hline \multirow{3}{*}{$\begin{array}{c}\text { Motivos de viagem } \\
(\%)\end{array}$} & Trabalho & 41,5 & 56,3 & $46,3 \uparrow$ & $61,3 \uparrow$ & $+0,2$ & Estável ( ) \\
\hline & Compras & 6,6 & 3,4 & $7,3 \uparrow$ & $4,1 \uparrow$ & 0,0 & Estável ( ) \\
\hline & Servir passageiro & 16,8 & 11,7 & $18,2 \uparrow$ & $12,4 \uparrow$ & $+0,7$ & Aumentou ( $\uparrow)$ \\
\hline \multicolumn{2}{|c|}{ Distância média de viagem $(\mathrm{km})$} & 3,9 & 5,1 & $4,6 \uparrow$ & $5,4 \uparrow$ & $-0,4$ & Diminuiu $(\downarrow)$ \\
\hline \multicolumn{2}{|c|}{$\%$ de viagens em horário fora de pico } & 52,5 & 51,8 & $50,9 \downarrow$ & $50,3 \downarrow$ & $-0,1$ & Estável ( ) \\
\hline \multicolumn{2}{|c|}{$\begin{array}{l}\text { Tempo total gasto diariamente em } \\
\text { deslocamentos (min) }\end{array}$} & 102,4 & 107,4 & $89,4 \downarrow$ & $91,4 \downarrow$ & $-3,0$ & Diminuiu $(\downarrow)$ \\
\hline \multicolumn{2}{|c|}{$\begin{array}{l}\text { Proporção de viagens de base } \\
\text { não-residencial }\end{array}$} & 11,9 & 12,3 & $9,9 \downarrow$ & $11,8 \downarrow$ & $+1,5$ & Aumentou ( $\uparrow)$ \\
\hline
\end{tabular}

\section{CONCLUSÕES}

Entende-se que o objetivo de caracterizar as diferenças no padrão de mobilidade de mulheres e homens em grandes cidades brasileiras, representando contextos regionais distintos, foi satisfatoriamente atendido. A partir dessa caracterização, foi possível avaliar um conjunto de hipóteses retiradas da literatura acerca dos efeitos da divisão sexual do trabalho. De modo geral, a análise das pesquisas OD domiciliares das três capitais selecionadas corrobora o que a literatura apresenta a respeito das desigualdades nos padrões de mobilidade entre gêneros. Questiona-se, entretanto, a capacidade dos indicadores escolhidos em representar bem o tempo total gasto com deslocamentos e o encadeamento de viagens, cujos resultados contradizem as hipóteses encontradas na literatura. Além de não ser obvia a explicação de por que tais valores são discrepantes, a evolução temporal dos dados para a cidade de São Paulo aponta para um aumento dessas diferenças. 
De forma complementar, a avaliação da variação temporal dos indicadores (limitada ao caso de São Paulo) descortina um outro conjunto de questões. Quanto aos valores absolutos dos indicadores para mulheres, os aumentos percebidos nos valores de alguns nem sempre são entendidos como benéficos (ex.: \% de viagens em hora de pico ou distancias médias de viagem), por remeterem a condições de mobilidade indesejáveis. Outros deixam dúvidas. Aumentar a participação de viagens por modos ativos pode ser um aspecto positivo, desde que resulte de uma escolha do usuário. Ao mesmo tempo, e no sentido oposto, a diminuição de certos indicadores pode ser vista como positiva em certos casos (ex.: diminuição da imobilidade) e negativa em outros (ex.: número médio de viagens por pessoa).

Além da variação absoluta de cada indicador ao longo do tempo, é importante também compreender como essas variações implicaram na redução ou não das desigualdades entre gêneros. Observou-se uma diminuição substancial dessas diferenças na distância média e no tempo total dos deslocamentos, assim como na proporção das viagens realizadas a pé. Por outro lado, verificou-se um aumento da desigualdade na taxa de imobilidade e na proporção de viagens com base não-domiciliar. Esse tipo de análise pode ser um primeiro passo na direção de reconhecer onde os problemas são mais agravados, o que pode funcionar como indicativo de priorizações de políticas públicas.

Apesar das contribuições fenomenológicas geradas a partir dessa análise de caracterização, deve-se reconhecer algumas limitações deste trabalho. A primeira está relacionada à seleção de apenas três cidades para análise comparativa, o que limita a generalização das suas conclusões em nível nacional. Essa limitação é mais agravada no caso da análise da evolução temporal da problemática, a qual foi realizada apenas para São Paulo. Além disso, as três cidades selecionadas, apesar de serem de grande porte, possuem níveis populacionais bastante distintos (Florianópolis com cerca de 500 mil habitantes, Salvador com cerca de 2,9 milhões e São Paulo com 12,3 milhões de habitantes), o que realça a necessidade de estudos em cidades de pequeno e médio portes, assim como análises adicionais para outras metrópoles brasileiras. Por último, o presente trabalho limitou-se à caracterização da problemática representada, sem envidar esforços mais sistematizados acerca das suas relações de causalidade. Dito isto, recomenda-se que trabalhos futuros busquem avançar em análises de diagnóstico das desigualdades de gênero na mobilidade urbana brasileira, investigando os mecanismos causais envolvidos e a sua heterogeneidade por gênero, preferencialmente com o uso do ferramental de inferência causal e modelos estatísticos multivariados.

\section{AGRADECIMENTOS}

Os autores deste trabalho agradecem ao CNPq pelo financiamento da pesquisa.

\section{REFERÊNCIAS BIBLIOGRÁFICAS}

Anand, A. e G. Tiwari (2006) A Gendered Perspective of the Shelter Transport Livelihood Link: The Case of Poor Women in Delhi. Transport Reviews, v. 26, n. 1, p. 63-80. DOI: 10.1080/01441640500175615

Bowman, J. L. e M. Ben-Akiva (1996) Activity Based Travel Forecasting. Conference of Activity Based Travel Forecasting, New Orleans, LA, USA.

Crane, R. (2007) Is There a Quiet Revolution in Women's Travel? Revisiting the Gender Gap in Commuting. Journal of the American Planning Association, vol. 73, n. 3, p. 298-316. DOI: 10.1080/01944360708977979

Hanson, S. (2010) Gender and Mobility: New Approaches for Informing Sustainability. Gender, Place and Culture, vol. 17, n. 1, p. 5-23. DOI: $10.1080 / 09663690903498225$

Hanson, S., e I. Johnston (1985) Gender Differences in Work-Trip Length: Explanations and Implications. Urban Geography, vol. 6, n. 3, p. 193-219. DOI: 10.2747/0272-3638.6.3.193

Kergoat, D. (2003) Divisão Sexual do Trabalho e Relações Sociais de Sexo. In: Emílio, M., M. Teixeira, M. Nobre e T. Godinho (eds.) Trabalho e Cidadania Ativa para as Mulheres. São Paulo, SP. 
Law, R. (1999) Beyond "Women and Transport": Towards New Geographies of Gender and Daily Mobility. Progress in Human Geography, vol. 23, n. 4, p. 567-588. DOI: 10.1191/030913299666161864

Lee, B. S. e J. F. McDonald (2003) Determinants of Commuting Time and Distance for Seoul Residents: The Impact of Family Status on the Commuting of Women. Urban Studies, vol. 40, n. 7, p. 1283-1302. DOI: 10.1080/0042098032000084604

Loukaitou-Sideris, A. (2010) What is Blocking her Path? Women, Mobility, and Security. Women's Issues in Transportation: Summary of the 4th International Conference, TRB, Irvine, CA, USA, v.1, p. 103-121. DOI: 10.17226/22901

Motte-Baumvol, B., \& Bonin, O. (2018). The spatial dimensions of immobility in France. Transportation, 45(5), 1231-1247. DOI: $10.1007 / \mathrm{s} 11116-017-9763-5$

Peters, D. (2002) Gender and Transport in Less Developed Countries: A Background Paper in Preparation for CSD-9. Paper Commissioned by UNED Forum, London, UK.

Pinheiro, L., N. O. Fontoura, A. C. Querino, A. Bonetti e W. Rosa (2009) Retrato das desigualdades de gênero e raça (3ª ed.). Instituto de Pesquisa Econômica Aplicada, São Paulo, SP.

Prashker, J., Y. Shiftan e P. Hershkovitch-Sarusi (2008) Residential Choice Location, Gender and the Commute Trip to Work in Tel Aviv. Journal of Transport Geography, vol. 16, n. 5, p. 332-341. DOI: 10.1016/j.jtrangeo.2008.02.001

Root, A. e L. Schinder (1999) Women, Motorization and the Environment. Transportation Research Part D: Transport and Environment, vol. 4, n. 5, p. 353-355. DOI: 10.1016/S1361-9209(99)00012-7

Rosenbloom, S. (1978) The Need for Study of Women's Travel Issues. Transportation, vol. 7, n. 4, p. 347-350. DOI: 10.1007/BF00168035

Rosenbloom, S. (2000) Trends in Women's Travel Patterns. Women's Travel Issues Second National Conference, TRB, Baltimore, MD, USA.

Rosenbloom, S. (2006) Understanding Women's and Men's Travel Patterns: The Research Challenge. Women's Issues in Transportation: Summary of the 1st International Conference, TRB, Chicago, IL, USA, v. 1, p. 7-28. DOI: 10.17226/23274

Rosenbloom, S. e M. Pleiss-Fraissard (2010) Women's Travel in Developed and Developing Countries: Two Versions of the Same Story? Women's Issues in Transportation: Summary of the 4th International Conference, TRB, Irvine, CA, USA, v.1, p. 63-77. DOI: 10.17226/22901

Sermons, M. W. e F. S. Koppelman (2001) Representing the Differences between Female and Male Commute Behavior in Residential Location Choice Models. Journal of Transport Geography, vol. 9, n. 2, p. 101-110. DOI: 10.1016/S09666923(00)00047-8

Srinivasan, S. e P. Rogers (2005) Travel Behavior of Low-Income Residents: Studying Two Contrasting Locations in the City of Chennai, India. Journal of Transport Geography, vol. 13, n. 3, p. 265-274. DOI: 10.1016/j.jtrangeo.2004.07.008

Svab, H. (2016) Evolução dos Padrões de Deslocamento na Região Metropolitana de São Paulo: A Necessidade de uma Análise de Gênero. Dissertação. Universidade de São Paulo, SP.

Venter, C., V. Vokolkova e J. Michalek (2007) Gender, residential location, and household travel: Empirical findings from lowincome urban settlements in Durban, South Africa. Transport Reviews, vol. 27, n. 6, p. 653-677. DOI: 10.1080/01441640701450627 